\title{
Spinal Cord Gray and White Matter Damage in Different Hereditary Spastic Paraplegia Subtypes
}

\author{
(D) K.R. Servelhere, (D)R.F. Casseb, (DF.D. de Lima, (D)T.J.R. Rezende, (DL.P. Ramalho, and (D) M.C. França Jr
}

\begin{abstract}
BACKGROUND AND PURPOSE: Spinal cord damage is a hallmark of hereditary spastic paraplegias, but it is still not clear whether specific subtypes of the disease have distinctive patterns of spinal cord gray (GM) and white (WM) matter involvement. We compared cervical cross-sectional GM and WM areas in patients with distinct hereditary spastic paraplegia subtypes. We also assessed whether these metrics correlated with clinical parameters.
\end{abstract}

MATERIALS AND METHODS: We analyzed 37 patients (17 men; mean age, 47.3 [SD, 16.5] years) and 21 healthy controls (7 men; mean age, 42.3 [SD, 13.2] years). There were 7 patients with spastic paraplegia type 3A (SPG3A), 12 with SPG4, 10 with SPG7, and 8 with SPG11. Image acquisition was performed on a 3T MR imaging scanner, and T2*-weighted 2D images were assessed by the Spinal Cord Toolbox. Statistical analyses were performed in SPSS using nonparametric tests and false discovery rate-corrected $P$ values $<.05$.

RESULTS: The mean disease duration for the hereditary spastic paraplegia group was 22.4 [SD, 13.8] years and the mean Spastic Paraplegia Rating Scale score was 22.8 [SD, 11.0]. We failed to identify spinal cord atrophy in SPG3A and SPG7. In contrast, we found abnormalities in patients with SPG4 and SPG11. Both subtypes had spinal cord GM and WM atrophy. SPG4 showed a strong inverse correlation between GM area and disease duration $(\rho=-0.903, P<.001)$.

CONCLUSIONS: Cervical spinal cord atrophy is found in some but not all hereditary spastic paraplegia subtypes. Spinal cord damage in SPG4 and 11 involves both GM and WM.

ABBREVIATIONS: CSA = cross sectional area; HSP = hereditary spastic paraplegia; $\mathrm{SC}=$ spinal cord; SCT = Spinal Cord Toolbox; SPG = spastic paraplegia; SPRS-BR $=$ Brazilian version of the Spastic Paraplegia Rating Scale

$\mathrm{H}$ ereditary spastic paraplegia (HSP) consists of a heterogeneous group of inherited neurodegenerative disorders in which the longest descending fibers of the spinal cord (SC) are the main target of damage. ${ }^{1-3}$ Clinically, patients present with spastic gait that can be associated with variable degrees of lower limb weakness as well as sensory deficits and bladder or bowel dysfunction. HSP can be inherited in every possible way, but autosomal dominant and recessive are the most common patterns of inheritance. ${ }^{4}$

Received May 22, 2020; accepted after revision October 4.

From the School of Medical Sciences (K.R.S., F.D.d.L. T.J.R.R., L.P.R., M.C.F.), University of Campinas, Campinas, Brazil; and Seaman Family MR Research Center (R.F.C.), University of Calgary, Calgary, Alberta, Canada.

This work was supported by the Coordenação de Aperfeiçoamento de Pessoal de Nivel Superior Foundation, project number PNPD20131798, and the Fundação de Amparo à Pesquisa do Estado de São Paulo 2013/07559-3.

Please address correspondence to Marcondes C. França, Jr, MD, PhD,

Neuromuscular Division, Department of Neurology, University of Campinas (UNICAMP). Rua Tessália de Vieira Camargo, 126, Cidade Universitária Zeferino Vaz. CEP 13083-887, Campinas, SP, Brasil; e-mail: mcfrancajr@uol.com.br

- Indicates open access to non-subscribers at www.ajnr.org

Indicates article with online supplemental data.

http://dx.doi.org/10.3174/ajnr.A7017
So far, $>70$ loci and 60 genes are linked to HSP, and the different genetic subtypes of the disease are spastic paraplegia (SPG) 1 to SPG80, corresponding to the chronologic order of gene description. ${ }^{4}$

Since the first postmortem descriptions in subjects with HSP, the corticospinal tract and the SC have been indicated as the key targets of damage in the disease. ${ }^{8,9}$ Studies using MR imaging have already shown atrophy of the SC in these patients. ${ }^{9-11}$ However, these studies had some important limitations. First, the sample sizes were small; thus, the authors lumped different HSP subtypes into a single group to compare with healthy controls. ${ }^{9-11}$ Second, MR imaging analyses failed to separate SC white (WM) and gray (GM) matter for individualized analyses. ${ }^{9-11}$

In this scenario, novel MR imaging acquisition protocols that allow quantifying GM and WM separately have been developed in the past decade. ${ }^{12}$ The Spinal Cord Toolbox (SCT; https:// spinalcordtoolbox.com/en/stable/), a robust tool capable of isolating GM and WM automatically, is a comprehensive and opensource software specifically designed to process SC MR imaging data and perform cord-specific quantification of cross- 
sectional areas across vertebral levels. ${ }^{13}$ This pipeline has high segmentation accuracy and acceptable intra- and interscanner reliability. ${ }^{13,14}$ The measurement of these segmented structures can contribute to identifying potential biomarkers for HSPs through understanding the pattern of SC damage. This might be helpful for diagnostic purposes and also to assess disease progression.

As far as we know, no studies approaching GM and WM separately in HSP have been performed. Therefore, this study used the SCT in a representative sample of genotype-specific HSP subtypes, aiming to compare cross-sectional cervical SC, GM, and WM areas. Furthermore, we verified whether these metrics correlated with clinical aspects such as age at onset, disease, and disease severity.

\section{MATERIALS AND METHODS \\ Subject Selection}

We initially recruited and scanned 55 patients and 23 healthy controls from 2018 to 2019 . We excluded 13 patients (4 with SPG15, 1 with SPG6, 3 with SPG72, 3 with SPG8, and 2 with SPG33) because these genotypes did not have large-enough sample sizes to enable comparisons. Furthermore, we excluded images from 5 patients (3 with SPG4, 1 with SPG11, and 1 with SPG3A) and 2 controls due to motion artifacts and poor segmentation. In the end, 37 patients with HSP (7 with SPG3A, 12 with SPG4, 10 with SPG7, and 8 with SPG11) with a mean age of 47.3 [SD, 16.5] years and 21 healthy controls with a mean age of 42.3 [SD, 13.2] years took part in the study. None of these remaining subjects had relevant disc disease or cord compression. All patients were followed at our neurogenetics outpatient clinic.

All patients were older than 18 years of age and had genotypes confirmed by molecular testing. This study was approved by the ethics committee of UNICAMP (CAAE 83241318.3.1001.5404) and have been performed in accordance with the ethical standards laid down in the 1964 Declaration of Helsinki and its later amendments. All subjects agreed to participate and signed an informed consent form before any study-related procedure.

\section{Clinical Parameters}

We recorded the age at onset of the first symptom and disease duration for all patients. Phenotypes were classified into pure and complicated. We used 3 clinical scales to quantify disease severity: the Brazilian version of the Spastic Paraplegia Rating Scale (SPRS-BR), the Ashworth scale, and the Medical Research Council Scale for Muscle Strength. ${ }^{15-17}$ The last 2 scales were applied only to the lower limb muscles. Clinical evaluations were performed by a specialized researcher on the same day that MR images were obtained.

\section{MRI Acquisition}

All images were acquired in an Achieva 3T scanner (Philips Healthcare) using a standard 16-channel neurovascular head coil. We obtained sagittal and axial standard T1 and T2 scans of the cervical SC to rule out incidental findings (eg, degenerative disc disease, cord compression). Routine brain MR images were also obtained to rule out incidental findings.
To locate the ROI within the cervical SC, we obtained sagittal T2-weighted images with the following parameters: FOV $=220 \times$ $220 \times 36 \mathrm{~mm}^{3}$; voxel size $=0.7 \times 0.7 \times 3 \mathrm{~mm}^{3}$; 11 slices; gap $=$ $0.3 \mathrm{~mm}$; flip angle $=90^{\circ} ; \mathrm{TR} / \mathrm{TE}=1075 / 120 \mathrm{~ms}$.

For quantitative analyses, we used the $\mathrm{T} 2{ }^{*}$-weighted $3 \mathrm{D}$ slabselective fast-field echo images acquired in the axial plane from $\mathrm{C} 2$ to $\mathrm{C} 4$ with an acquisition time of 4 minutes 30 seconds $\left(\mathrm{FOV}=200 \times 153 \times 49 \mathrm{~mm}^{3}\right.$; voxel size $=0.8 \times 0.8 \times 3 \mathrm{~mm}^{3}$; matrix $=252 \times 191 ; 15$ slices; gap $=0.3 \mathrm{~mm} ; 4$ echoes; flip angle $=28^{\circ} ; \mathrm{TR} / \mathrm{TE}=700 / 6.7 \mathrm{~ms}$ ).

\section{MRI Analysis}

We used the SCT pipeline, Version 4.0.1, to obtain SC metrics. ${ }^{13}$ In brief, we followed some processing steps. Initially, we obtained the automatic segmentation of the cross-sectional total SC and GM areas using deep learning algorithms, followed by manual correction of the segmentation if necessary. This initial step enabled the generation of the WM area as the difference between cross sectional area (CSA)-GM areas. Next, the vertebral levels $\mathrm{C} 2, \mathrm{C} 3$, and C4 were automatically identified. Afterward, we proceeded to the registering of the $\mathrm{T}^{*}$ images to the PAM50 Template (https://spinalcordtoolbox.com/en/stable/overview/ concepts/pam50.html) by means of linear and/or nonlinear algorithms. Last, this template was warped to match the subject imaging, so that the mean total CSA and GM area for C2, C3, and $\mathrm{C} 4$ vertebrae can be computed. These measures are corrected for the curvature of the spine using the angle of the section with the SC centerline. Fifteen slices for each SC level were considered, and a mean value was estimated. Figure 1 shows examples of SC segmentation obtained using the SCT.

\section{Statistical Analysis}

We compared the entire HSP group as well as the subgroups defined according to the genetic test (SPG3A, SPG4, SPG7, and SPG11) against the entire control group $(n=21)$. Considering the relatively small sample size of each HSP subgroup, we used a nonparametric test (Mann-Whitney) to perform between-group comparisons of the CSA, GM, and WM areas at each spinal level. We applied the Benjamini-Hochberg correction to control for the false discovery rate in these analyses.

Correlation analyses were assessed separately within each HSP subtype using Spearman coefficients $(\rho)$. Again, we investigated whether CSA, GM, and WM measures correlated with clinical parameters, such as age at onset, disease severity, and disease duration. Because this investigation was exploratory, we decided not to correct for multiple correlations. Although we have an apriori hypotheses for each correlation, we accepted a more conservative 2 -tailed $P$ value. We set $P<.05$ for all analyses.

\section{RESULTS}

There were 17 men in the patient group (45.9\%) and 7 men $(33.3 \%)$ in the control group. There was no significant difference regarding age between groups (patients versus healthy controls). Patients had a mean disease duration of 22.4 [SD, 13.8] years, and the mean SPRS-BR score was 22.8 [SD, 11.0]. There were 17 patients with complicated HSP (8 with SPG11 and 9 with SPG7) and 20 with pure HSP (7 with SPG3A, 12 with SPG4, and 1 with 
SPG7). When we considered those with complicated phenotypes, all subjects with SPG11 had neurophysiologic signs of chronic lower motor neuron involvement (including the arms), and all except one had cognitive decline. The 9 patients with SPG7 had cerebellar ataxia. The patients with pure HSP only had lowerlimb pyramidal and sensory manifestations. Ten patients were wheelchair-bound, 12 used canes, and 3 needed some assistance to walk. Demographic data of each HSP subtype are presented in the Table.

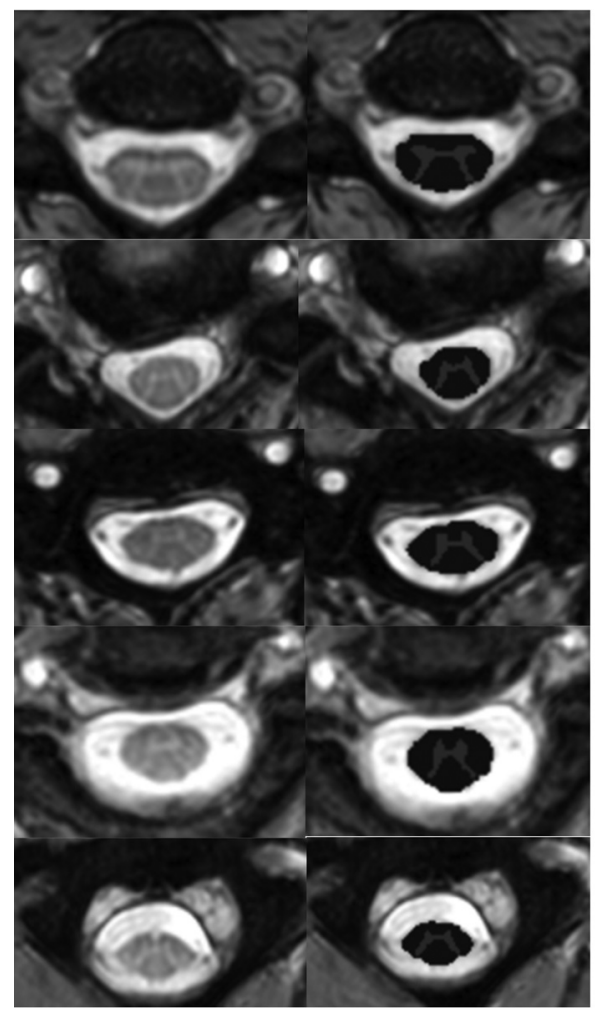

HEALTHY CONTROLS

HSP-SPG3A

HSP-SPG4

HSP-SPG7

HSP-SPG11

FIG 1. Axial T2* slices of the cervical spinal cord in a healthy control (upper row) and patients with HSP-SPG3A, HSP-SPG4, HSP-SPG7, and HSP-SPGI1 (lower rows). In each case, the original image is shown in the left column, whereas the segmented white (dark gray) as well as the gray matter (light gray) cross-sectional areas are shown in the right column.

Demographic and clinical data from genotype-specific patients with HSP included in this study ${ }^{a}$

\begin{tabular}{lcccc}
\hline \multicolumn{1}{c}{ Parameter } & SPG3A & SPG4 & \multicolumn{1}{c}{ SPG7 } & \multicolumn{1}{c}{ SPG11 } \\
\hline Sample (No.) & 7 & 12 & 10 & 8 \\
Sex (M/F) & $2: 5$ & $6: 6$ & $5: 5$ & $4: 4$ \\
Age (yr) & $37.0(12.0)$ & $57.0(12.5)$ & $63.0(10.0)$ & $29.0(6.0)$ \\
Age at onset (yr) & $0(3.5)$ & $37.5(8.5)$ & $35.0(10.0)$ & $17.5(3.5)$ \\
Disease duration (yr) & $33.0(10.2)$ & $21.0(13.0)$ & $27.0(24.0)$ & $10.0(6.0)$ \\
SPRS-BR & $21.0(18.0)$ & $18.5(9.0)$ & $22.5(23.0)$ & $24.0(9.5)$ \\
Leg spasticity & $1(0-2)$ & $1(0-2)$ & $1(0-2)$ & $1(0-2)$ \\
Leg muscle strength & $4(3-5)$ & $4(4-5)$ & $5(4-5)$ & $4(4-5)$ \\
\hline
\end{tabular}

a Parameters are expressed using median (interquartile range). Muscle spasticity and strength were assessed in the following lower limb muscles: thigh adductors and abductors, quadriceps, hamstrings, ankle plantar flexors and dorsal flexors using the Ashworth scale and the Medical Research Council Scale for Muscle Strength respectively. Results shown in the table represent an average value from all muscles tested in each patient.

\section{MRI Group Comparison (Patients versus Healthy Controls)}

Considering the entire HSP group, all measures (CSA, GM, and $\mathrm{WM}$ ) at all levels were significantly smaller than in the control group (Online Supplemental Data). There was a significant difference in terms of CSA only for HSP-SPG4 and HSP-SPG11 (Online Supplemental Data and Fig 2). All 3 evaluated slices presented significant area reduction for both genetic subtypes. The pattern of GM and WM damage was also similar in HSP-SPG4 and HSP-SPG11 (Online Supplemental Data and Fig 2). In both groups, we found GM as well as WM area reduction in comparison with healthy controls.

CSA, GM, and WM areas were not different between controls and the HSP-SPG3A and SPG7 groups at any spinal level.

\section{Correlations (Clinical Parameters versus SC Areas)}

We assessed correlations using the Spearman rank correlation coefficient. Considering that only HSP-SPG4 and SPG11 had significant SC morphometric changes, we decided to explore potential clinical correlates (age at onset, disease duration, and disease severity) for only those 2 subgroups. None of these parameters correlated with imaging findings in the HSP-SPG11 group. In contrast, we found significant correlations in the HSP-SPG4 group: 1) CSA at $\mathrm{C} 4$ had a direct correlation with the age at onset ( $\rho=0.753, P=.005) ; 2$ ) the GM area at C3 had a direct correlation with age at onset $(\rho=0.706, P=.01)$ and a negative correlation with disease severity, expressed by SPRS-BR scores ( $\rho=-$ $0.630, P=.03$ ); and 3) GM area at $\mathrm{C} 4$ had a positive correlation with age at onset $(\rho=0.754, P=.01)$, but a negative correlation with disease duration $(\rho=-0.903, P<.001)$ and disease severity ( $\rho=-0.866, P=.001)$.

As a second step, for each HSP group, we performed correlation analyses of SC areas and disease severity, controlling for age, sex, and disease duration as covariates, but none of the results reached the significance threshold $(P<.05)$.

\section{DISCUSSION}

This study focused on the comparison of cervical SC, GM, and WM areas in 4 genotype-specific HSP groups and healthy controls. Currently, there are some pipelines available for SC segmentation, ${ }^{18,19}$ but we chose the SCT because it is highly reproducible and less affected by artifacts. ${ }^{12-14}$ Then, we were able to show, for the first time, that cervical SC atrophy is found in some but not all HSP subtypes. Moreover, SC morphometric changes in HSP-SPG4 and SPG11 extend to both GM and WM. SC WM involvement in HSPSPG4 and SPG11 is expected because of the involvement of the corticospinal tract, which has been shown in previous pathologic reports in both diseases. ${ }^{20,21}$ It is rather probable that corticospinal tract axonal loss and gliosis are the pathologic correlates of the atrophy we found using MR imaging. In addition to upper motor neuron damage, HSP-SPG11 is also characterized by lower motor neuron dysfunction. 

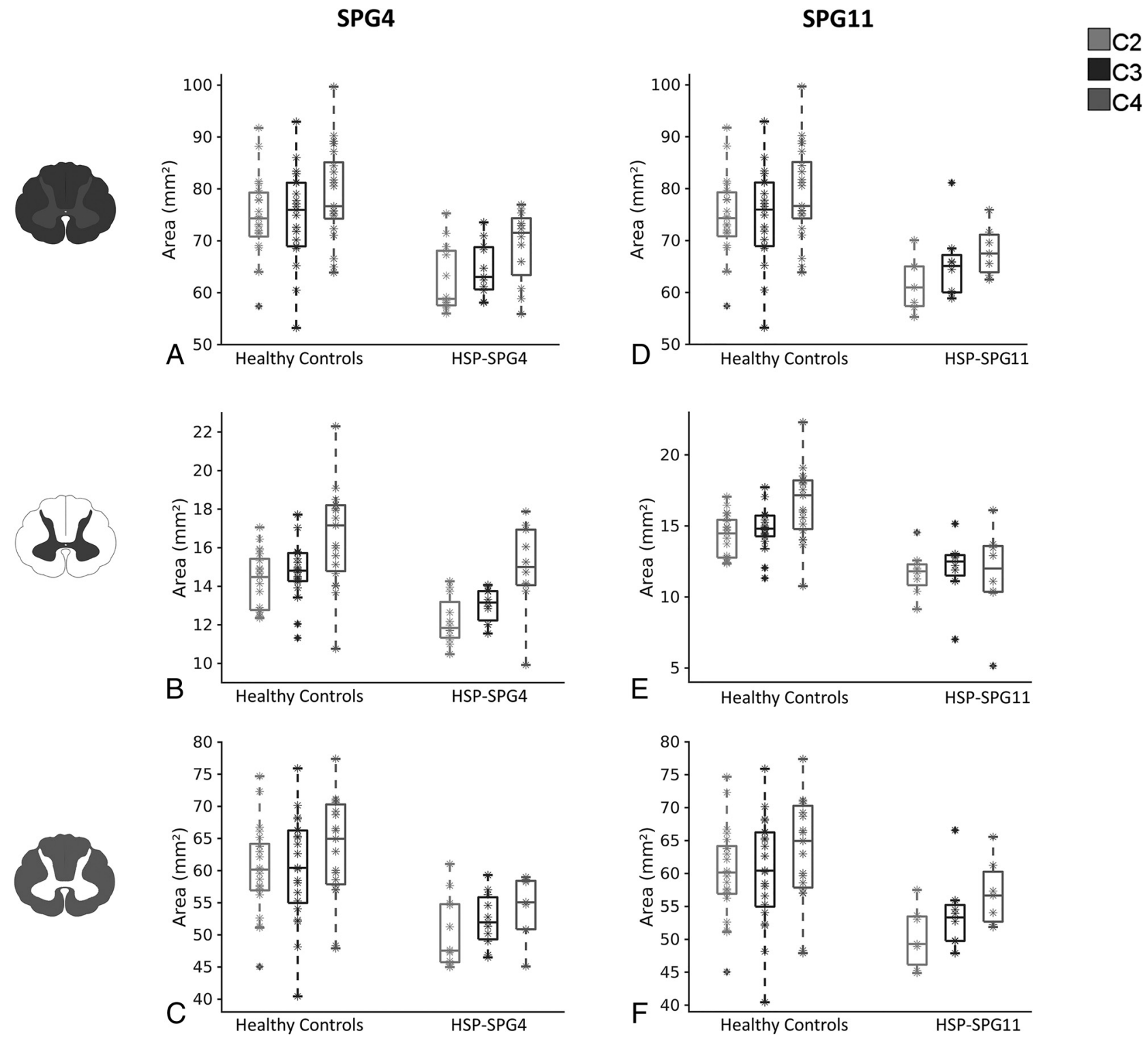

FIG 2. Box-and-whisker plots showing the distribution of the SC (upper row), GM (middle row), and WM (lower row) areas of patients with HSP-SPG4 versus healthy controls (left side) and patients with HSP-SPG11 versus healthy controls (right side) along cervical levels C2, C3, and C4.

Some patients actually present with a phenotype resembling juvenile amyotrophic lateral sclerosis. ${ }^{21,22}$ Faber et al, ${ }^{22}$ found almost universal lower motor neuron disease in a cohort of 25 subjects with HSP-SPG11. All patients with SPG11 herein included indeed had signs of anterior horn damage (including the arms). In this scenario, we hypothesize that reduced GM area in this genetic subtype could be related to the loss of motor neurons in the ventral horns of the SC. ${ }^{21}$ The explanation for SC GM atrophy in subjects with HSP-SPG4 must not be the same because these patients do not have lower motor neuron disease in general. ${ }^{23}$ In our cohort of HSP-SPG4, none of the subjects had clinical lower motor neuron signs, but only a few of them underwent nerve conduction studies and electromyography. Then, it is possible that we may have missed subclinical lower motoneuronal involvement. Nevertheless, we believe that loss of other neuronal populations within SC GM, such as those located in the sensory lamina of Rexed, is a more reasonable explanation for GM atrophy. This last hypothesis deserves further investigation in pathologic studies.

Another interesting finding in this study was the striking correlation between SC metrics and clinical disability in SPG4. In this subgroup, SC GM but not WM area at C4 had an extremely high and statistically significant association with both disease duration and severity. This was somewhat unexpected but indicates that damage to neuronal circuits within the SC GM is relevant to the disease. In the face of these findings, one should no longer consider this HSP subtype as a "pure" distal motor axonopathy. In addition, our results highlight the importance of pursuing SC segmentation into GM and WM in the context of HSPs. Separate analyses of GM and WM, rather than just looking at the entire SC area, might provide more interesting insights, not only into the biology of these diseases but also into potential biomarkers for clinical use. Some previous studies indeed failed to identify the clinical correlates of SC damage, possibly because they used 
this whole SC measurement approach. ${ }^{9,10}$ In the near future with technical MR imaging advancements, it is possible that deeper segmentation of the SC, for instance, determining the area of the anterior and posterior horns as well the dorsal columns, will help us even more in the understanding of HSP. ${ }^{24,25}$ Other metabolic and genetic SC disorders may also benefit from a similar approach using quantitative GM and WM evaluation. ${ }^{26}$

We failed to show the same relevant associations for subtypes HSP-SPG7 and SPG11. This could be due to a ceiling effect of SPRS-BR for these 2 subtypes. Indeed, patients with SPG7 and SPG11 were the most disabled (many of them already wheelchair-bound). In late disease stages, the scale fails to capture clinical deterioration (even though neurodegeneration was still taking place), and this may have interfered with the correlation analyses. $^{27}$ The assessment of cohorts in the early stages of SPG7 and SPG11 would help to clarify this point.

We were not able to find SC atrophy in the HSP-SPG3A and SPG7 groups. This may indicate that SC involvement in these subtypes does not lead to macroscopic abnormalities. Only microstructural abnormalities would be present and could only be uncovered with other imaging techniques, such as DTI. $^{25}$ An alternative explanation is that SC volumetric reduction actually takes place but is mild. In this scenario, our sample size could have been underpowered to detect such small differences. This is sometimes an issue in single-center studies dealing with rare diseases such as HSP. To overcome these limitations, multicentric studies using a multimodal SC MR imaging approach in a longitudinal setting would be important to enable the recruitment of more patients, including HSP subtypes not explored here. Additional levels of the SC, such as the thoracic region, were not evaluated in our study and should be assessed in these future studies as well.

\section{CONCLUSIONS}

We were able to show that HSP types 4 and 11 have SC atrophy involving both GM and WM. GM SC morphometry correlated with clinical parameters in HSP-SPG4 and might be a useful biomarker to track disease progression in this condition, but longitudinal studies should now be performed to validate these findings.

\section{ACKNOWLEDGMENTS}

This study was approved by the ethics committee of University of Campinas (CAAE 83241318.3.1001.5404) and has been performed in accordance with the ethical standards laid down in the 1964 Declaration of Helsinki and its later amendments.

Disclosures: Katiane R. Servelhere-RELATED: Grant: Coordenação de Aperfeiçoamento de Pessoal de Nível Superior Foundation, Comments: scholarship from Coordenação de Aperfeiçoamento de Pessoal de Nível Superior Foundation; Support for Travel to Meetings for the Study or Other Purposes: Coordenação de Aperfeiçoamento de Pessoal de Nível Superior Foundation, Comments: scholarship from Coordenação de Aperfeiçoamento de Pessoal de Nível Superior Foundation. Fabricio Diniz de Lima—RELATED: Grant: Fundação de Amparo à Pesquisa do Estado de São Paulo.* Thiago J. de Rezende-UNRELATED: Grants/Grants Pending: Fundação de Amparo à Pesquisa do Estado de São Paulo, Comments: postdoctorate scholarship. Marcondes Cavalcante França, Jr-RELATED: Grant: Fundação de Amparo à Pesquisa do Estado de São Paulo, Comments: Fundação de Amparo à
Pesquisa do Estado de São Paulo is a governmental agency that supports research in Brazil.* *Money paid to the institution.

\section{REFERENCES}

1. Parodi L, Fenu S, Stevanin G, et al. Hereditary spastic paraplegia: more than an upper motor neuron disease. Rev Neurol (Paris) 2017;173:352-60 CrossRef Medline

2. Faber I, Pereira ER, Martinez AR, et al. Hereditary spastic paraplegia from 1880 to 2017: an historical review. Arq Neuropsiquiatr 2017;75:813-18 CrossRef Medline

3. Parodi L, Coarelli G, Stevanin G, et al. Hereditary ataxias and paraparesias: clinical and genetic update. Curr Opin Neurol 2018;31:46271 CrossRef Medline

4. Shribman S, Reid E, Crosby AH, et al. Hereditary spastic paraplegia: from diagnosis to emerging therapeutic approaches. Lancet Neurol 2019;18:1136-46 CrossRef

5. Strümpell A. Ueber eine bestimmte Form der primaren combinirten Systemerkrankung des Rückenmarks. Archiv für Psychiatrie und Nervenkrankheiten 1886;17:217-38 CrossRef

6. Strümpell A. Die primare Seitenstrangsklerose (spastische Spinalparalyse). Deutsche Zeitschrift für Nervenheilkunde 1904;27:291-339 CrossRef

7. DeLuca GC, Ebers GC, Esiri MM. The extent of axonal loss in the long tracts in hereditary spastic paraplegia. Neuropathol Appl Neurobiol 2004;30:576-84 CrossRef Medline

8. List J, Kohl Z, Winkler J, et al. Ascending axonal degeneration of the corticospinal tract in pure hereditary spastic paraplegia: a cross-sectional DTI study. Brain Sci 2019;9:268 CrossRef Medline

9. Hedera P, Eldevik OP, Maly P, et al. Spinal cord magnetic resonance imaging in autosomal dominant hereditary spastic paraplegia. Neuroradiol 2005;47:730-34 CrossRef Medline

10. Sperfeld AD, Baumgartner A, Kassubek J. Magnetic resonance investigation of the upper spinal cord in pure and complicated hereditary spastic paraparesis. Eur Neurol 2005;54:181-85 CrossRef Medline

11. Agosta F, Scarlato M, Spinelli EG, et al. Hereditary spastic paraplegia: beyond clinical phenotypes toward a unified pattern of central nervous system damage. Radiology 2015;276:207-18 CrossRef Medline

12. Prados F, Ashburner J, Blaiotta C, et al. Spinal cord grey matter segmentation challenge. Neuroimage 2017;152:312-29 CrossRef Medline

13. De Leener B, Lévy S, Dupont SM, et al. SCT: Spinal Cord Toolbox, an open-source software for processing spinal cord MRI data. Neuroimage 2017;145:24-43 CrossRef Medline

14. Papinutto N, Henry RG. Evaluation of intra- and interscanner reliability of MRI protocols for spinal cord gray matter and total cross-sectional area measurements. J Magn Reson Imaging 2019;49:1078-90 CrossRef

15. Servelhere KR, Faber I, Coan AC, et al. Translation and validation into Brazilian Portuguese of the Spastic Paraplegia Rating Scale (SPRS). Arq Neuropsiquiatr 2016;74:489-94 CrossRef Medline

16. Bohannon RW, Smith MB. Interrater reliability of a modified Ashworth scale of muscle spasticity. Phys Ther 1987;67:206-07 CrossRef Medline

17. Compston A. Aids to the investigation of peripheral nerve injuries. Medical Research Council: Nerve Injuries Research Committee. His Majesty's Stationery Office: 1942; pp. 48 (iii) and 74 figures and 7 diagrams; with aids to the examination of the peripheral nervous system. By Michael O'Brien for the Guarantors of Brain. Saunders Elsevier: 2010; pp. [8] 64 and 94 Figures. Brain 2010;133:2838-44 CrossRef Medline

18. Datta E, Papinutto N, Schlaeger R, et al. Gray matter segmentation of the spinal cord with active contours in MR images. Neuroimage 2017;147:788-99 CrossRef Medline

19. Papinutto N, Schlaeger R, Panara V, et al. 2D phase-sensitive inversion recovery imaging to measure in vivo spinal cord gray and 
white matter areas in clinically feasible acquisition times. J Magn Reson Imaging 2015;42:698-708 CrossRef Medline

20. White KD, Ince PG, Lusher M, et al. Clinical and pathologic findings in hereditary spastic paraparesis with spastin mutation. Neurology 2000;55:89-94 CrossRef Medline

21. Denora PS, Smets K, Zolfanelli F, et al. Motor neuron degeneration in spastic paraplegia 11 mimics amyotrophic lateral sclerosis lesions. Brain 2016;139:1723-34 CrossRef Medline

22. Faber I, Martinez AR, de Rezende TJR, et al. SPG11 mutations cause widespread white matter and basal ganglia abnormalities, but restricted cortical damage. Neuroimage Clin 2018;19:848-57 CrossRef Medline

23. Parodi L, Rydning SL, Tallaksen C, et al. Spastic paraplegia 4. GeneReviews [Internet] 2003 April 17. [Updated 2019 Jun 13]
24. Paquin ME, EL Mendili MM, Gros C, et al. Spinal cord gray matter atrophy in amyotrophic lateral sclerosis. AJNR Am J Neuroradiol 2018;39:184-92 CrossRef Medline

25. Samson RS, Lévy S, Schneider T, et al. Zoom or Non-Zoom? Assessing spinal cord diffusion tensor imaging protocols for multi-centre studies. PLoS One 2016;11:e0155557 CrossRef Medline

26. Marelli C, Salsano E, Politi LS, et al. Spinal cord involvement in adult-onset metabolic and genetic diseases. J Neurol Neurosurg Psychiatry 2019;90:211-18 CrossRef Medline

27. Schüle R, Holland-Letz T, Klimpe S, et al. The Spastic Paraplegia Rating Scale (SPRS): a reliable and valid measure of disease severity. Neurology 2006;67:430-34 CrossRef Medline 